\title{
Some elliptic system and reduction method
}

\author{
Tacksun Jung $^{1 *}$ and Q-Heung Choi ${ }^{2}$
}

"Correspondence:

tsjung@kunsan.ac.kr

${ }^{1}$ Department of Mathematics,

Kunsan National University, Kunsan,

573-701, Korea

Full list of author information is

available at the end of the article

\section{Abstract}

We get a theorem which shows the existence of at least three solutions for some elliptic system with Dirichlet boundary condition. We obtain this result by using the finite dimensional reduction method for the dimension of the system which reduces the infinite dimensional problem to the finite dimensional one. We also use critical point theory on the reduced finite dimensional subspace.

MSC: 35J50; 35J55

Keywords: elliptic system; reduction method; (PS) condition; critical point theory; variational method

\section{Introduction}

In this paper we are concerned with multiple solutions for a class of systems of elliptic equations with Dirichlet boundary condition

$$
\begin{aligned}
& -\Delta u_{1}=F_{u_{1}}\left(x, u_{1}, \ldots, u_{n}\right) \quad \text { in } \Omega, \\
& -\Delta u_{2}=F_{u_{2}}\left(x, u_{1}, \ldots, u_{n}\right) \quad \text { in } \Omega, \\
& \vdots \\
& -\Delta u_{n}=F_{u_{n}}\left(x, u_{1}, \ldots, u_{n}\right) \quad \text { in } \Omega, \\
& u_{i}(x)=0, \quad i=1, \ldots, n, \text { on } \partial \Omega,
\end{aligned}
$$

where $\Omega$ is a bounded subset of $R^{n}$ with smooth boundary $\partial \Omega, n \geq 3, u_{i}(x) \in W_{0}^{1,2}(\Omega)$, $F: R^{n} \times R^{n} \rightarrow R$ is a $C^{2}$ function such that $F(x, \theta)=0, \theta=(0, \ldots, 0)$ and $F_{u_{i}}\left(x, u_{1}, \ldots, u_{n}\right)=$ $\frac{\partial F\left(x, u_{1}, \ldots, u_{n}\right)}{\partial u_{i}}, i=1, \ldots, n$. Let $U=\left(u_{1}, \ldots, u_{n}\right)$ and $\|\cdot\|_{R^{n}}$ denote the Euclidean norm in $R^{n}$. Let us define

$$
d_{U} F(x, U)=F_{U}(x, U)=\operatorname{grad}_{U} F(x, U)=\left(F_{u_{1}}\left(x, u_{1}, \ldots, u_{n}\right), \ldots, F_{u_{n}}\left(x, u_{1}, \ldots, u_{n}\right)\right)
$$

and

$$
d_{U}^{2} F(U) \cdot U=d\left(F_{U}(x, U)\right) \cdot U \quad \forall U \in E
$$

Let $\lambda_{1}<\lambda_{2} \leq \cdots \leq \lambda_{k} \leq \cdots$ be eigenvalues of the eigenvalue problem $-\Delta u=\lambda u$ in $\Omega, u=0$ on $\partial \Omega$, and $\phi_{k}$ be an eigenfunction belonging to the eigenvalue $\lambda_{k}, k \geq 1$.

We assume that $F$ satisfies the following conditions:

@ 2015 Jung and Choi; licensee Springer. This is an Open Access article distributed under the terms of the Creative Commons Attribution License (http://creativecommons.org/licenses/by/4.0), which permits unrestricted use, distribution, and reproduction in any medium, provided the original work is properly credited. 
(F1) $F \in C^{2}\left(R^{n} \times R^{n}, R\right), F(x, \theta)=0, F_{U}(x, \theta)=\theta, x \in \Omega, \theta=(0, \ldots, 0)$.

(F2) There exist constants $\alpha$ and $\beta$ ( $\alpha, \beta$ are not eigenvalues of the elliptic eigenvalue problem) such that $\alpha<\beta$ and

$$
\alpha I \leq d_{U}^{2} F(x, U) \leq \beta I \quad \forall(x, U) \in R^{n} \times R^{n}
$$

and there exists $k \in N^{*}$ such that $\alpha I<\lambda_{k} I<d_{U}^{2} F(x, U)<\lambda_{k+1} I<\beta I$ for every $U$, where $I$ is the $n \times n$ identity matrix.

(F3) There exist eigenvalues $\lambda_{h+1}, \ldots, \lambda_{h+m}$ such that

$$
\lambda_{h}<\alpha<\lambda_{h+1}<\cdots<\lambda_{h+m}<\beta<\lambda_{h+m+1}
$$

where $h \geq 1, m \geq 1$.

(F4) There exist $\gamma$ and $C$ such that $\lambda_{h+m}<\gamma<\beta$ and

$$
F(x, U) \geq \frac{1}{2} \gamma\|U\|_{R^{n}}^{2}-C, \quad \forall(x, U) \in R^{n} \times R^{n} .
$$

Some papers of Lee [1-4] concerning the semilinear elliptic system and some papers of the other several authors $[5,6]$ have treated the system of this like nonlinear elliptic equations. Some papers of Chang [7] and Choi and Jung [8] considered the existence and multiplicity of weak solutions for nonlinear boundary value problems with asymptotically linear term. The authors obtained some results for those problems by approaching the variational method, critical point theory and the topological method.

Let $W_{0}^{1,2}(\Omega, R)$ be the Sobolev space with the norm

$$
\|u\|_{W_{0}^{1,2}(\Omega, R)}^{2}=\int_{\Omega}|\nabla u|^{2} d x \quad \text { for } u \in W_{0}^{1,2}(\Omega)
$$

and the scalar product

$$
(u, v)_{W_{0}^{1,2}(\Omega, R)}=(\nabla u, \nabla v)_{L^{2}(\Omega, R)}
$$

Let $E$ be a cartesian product of the Sobolev spaces $W_{0}^{1,2}(\Omega, R)$, i.e.,

$$
E=W_{0}^{1,2}(\Omega, R) \times \cdots \times W_{0}^{1,2}(\Omega, R) .
$$

We endow the Hilbert space $E$ with the norm

$$
\|U\|_{E}^{2}=\sum_{i=1}^{n}\left\|u_{i}\right\|_{W_{0}^{1,2}(\Omega)}^{2}
$$

where $\left\|u_{i}\right\|_{W_{0}^{1,2}(\Omega, R)}^{2}=\int_{\Omega}\left|\nabla u_{i}(x)\right|^{2} d x$. From now on we shall denote by $W_{0}^{1,2}(\Omega)$ instead of $W_{0}^{1,2}(\Omega, R)$.

System (1.1) can be rewritten by

$$
\begin{aligned}
& -\Delta U=\operatorname{grad}_{U} F(x, U) \text { in } \Omega, \\
& U=\theta \text { on } \partial \Omega,
\end{aligned}
$$


where $-\Delta U=\left(-\Delta u_{1}, \ldots,-\Delta u_{n}\right)$ and $\theta=(0, \ldots, 0)$. In this paper we are looking for weak solutions of system (1.1) in $E$, that is, $U=\left(u_{1}, \ldots, u_{n}\right) \in E$ such that

$$
\int_{\Omega}[-\Delta U \cdot V] d x-\int_{\Omega} F_{U}(x, U) \cdot V=0 \quad \text { for all } V \in E
$$

Our main result is the following.

Theorem 1.1 Assume that F satisfies conditions (F1)-(F4). Then system (1.1) has at least three nontrivial weak solutions.

The proof of Theorem 1.1 is organized as follows: We approach the variational method and use the finite dimensional reduction method for the dimension of the system, which reduces the infinite dimensional problem to the finite dimensional one, and we get critical points of the functional on the infinite dimensional space $E$ from that of the reduced functional on the finite dimensional subspace of $E$. We also use critical point theory on the reduced finite dimensional subspace. In Section 2, we approach the variational method and the reduction method. We show that the reduced functional satisfies the (PS) condition. In Section 3, we prove Theorem 1.1.

\section{Reduction approach}

We assume that $F \in C^{2}\left(R^{n} \times R^{n}, R\right), F(x, \theta)=0, F_{U}(x, \theta)=\theta, \theta=(0, \ldots, 0)$ and there exist constants $\alpha$ and $\beta$ ( $\alpha, \beta$ are not eigenvalues of the elliptic eigenvalue problem) such that $\alpha<\beta$ and

$$
\alpha I \leq d_{U}^{2} F(x, U) \leq \beta I \quad \forall(x, U) \in R^{n} \times R^{n},
$$

and there exists $k \in N^{*}$ such that $\alpha I<\lambda_{k} I<d_{U}^{2} F(x, U)<\lambda_{k+1} I<\beta I$ for every $U$, where $U=\left(u_{1}, \ldots, u_{n}\right)$ and there exist eigenvalues $\lambda_{h+1}, \ldots, \lambda_{h+m}$ such that

$$
\lambda_{h}<\alpha<\lambda_{h+1}<\cdots<\lambda_{h+m}<\beta<\lambda_{h+m+1},
$$

where $h \geq 1, m \geq 1$.

Lemma 2.1 Let $F_{u_{i}}(x, U) \in L^{2}(\Omega), U=\left(u_{1}, \ldots, u_{i}, \ldots, u_{n}\right), i=1, \ldots, n$. Then all the solutions of

$$
-\Delta U=\operatorname{grad}_{U} F(x, U)
$$

belong to $E$.

Proof Let $F_{u_{i}}(x, U) \in L^{2}(\Omega)$. We note that $\left\{\lambda_{n}:\left|\lambda_{n}\right|<|c|\right\}$ is finite. Then $F_{u_{i}}\left(x, u_{1}, \ldots, u_{n}\right) \in$ $L^{2}(\Omega), i=1, \ldots, n$, can be expressed by

$$
F_{u_{i}}\left(x, u_{1}, \ldots, u_{n}\right)=\sum_{k=1}^{\infty} h_{k} \phi_{k}, \quad \sum_{k=1}^{\infty} h_{k}^{2}<\infty \quad \text { for each } i=1, \ldots, n .
$$


Then

$$
(-\Delta)^{-1} F_{u_{i}}\left(x, u_{1}, \ldots, u_{n}\right)=\sum \frac{1}{\lambda_{k}} h_{k} \phi_{k} .
$$

Hence we have the inequality

$$
\left\|(-\Delta)^{-1} F_{u_{i}}\left(x, u_{1}, \ldots, u_{n}\right)\right\|_{W_{0}^{1,2}(\Omega)}^{2}=\sum \lambda_{k} \frac{1}{\lambda_{k}^{2}} h_{k}^{2} \leq \sum h_{k}^{2}<\infty
$$

which means that

$$
\left\|(-\Delta)^{-1} F_{u_{i}}\left(x, u_{1}, \ldots, u_{n}\right)\right\|_{W_{0}^{1,2}(\Omega)} \leq\left\|F_{u_{i}}\left(x, u_{1}, \ldots, u_{n}\right)\right\|_{L^{2}(\Omega)}<\infty
$$

so $\left\|u_{i}\right\|_{W_{0}^{1,2}(\Omega)}<\infty$. Thus

$$
\|U\|_{E}=\left(\sum_{i=1}^{n}\left\|u_{i}\right\|_{W_{0}^{1,2}(\Omega)}\right)^{\frac{1}{2}}<\infty .
$$

By the following Lemma 2.2, weak solutions of system (1.1) coincide with critical points of the associated functional $I$

$$
\begin{aligned}
& I \in C^{1,1}(E, R), \\
& I(U)=\int_{\Omega}\left[\frac{1}{2}|\nabla U|^{2}-F(x, U)\right] d x,
\end{aligned}
$$

where $U=\left(u_{1}, \ldots, u_{n}\right)$ and $\int_{\Omega}|\nabla U|^{2} d x=\sum_{i=1}^{n} \int_{\Omega}\left|\nabla u_{i}\right|^{2} d x, n \geq 1$.

Lemma 2.2 Assume that F satisfies conditions (F1)-(F4). Then the functional I(U) is continuous, Fréchet differentiable with Fréchet derivative

$$
D I(U) \cdot V=\int_{\Omega}\left[(-\Delta U) \cdot V-F_{U}(x, U) \cdot V\right] d x
$$

Moreover, $D I \in C$. That is, $I \in C^{1}$.

Proof First we shall prove that $I(U)$ is continuous. For $U, V \in E$,

$$
\begin{aligned}
|I(U+V)-I(U)|= & \mid \frac{1}{2} \int_{\Omega}(-\Delta U-\Delta V) \cdot(U+V) d x-\int_{\Omega} F(x, U+V) d x \\
& -\frac{1}{2} \int_{\Omega}(-\Delta U) \cdot U d x+\int_{\Omega} F(x, U) d x \mid \\
= & \mid \frac{1}{2} \int_{\Omega}(-\Delta U \cdot V-\Delta V \cdot U-\Delta V \cdot V) d x \\
& -\int_{\Omega}(F(x, U+V)-F(x, U)) d x \mid .
\end{aligned}
$$

We have

$$
\left|\int_{\Omega}[F(x, U+V)-F(x, U)] d x\right| \leq\left|\int_{\Omega}\left[F_{U}(x, U) \cdot V+O\left(\|V\|_{E}\right)\right] d x\right|=O\left(\|V\|_{E}\right) .
$$


Thus we have

$$
\begin{aligned}
& |I(U+V)-I(U)|=O\left(\|V\|_{E}\right), \\
& |I(U+V)-I(U)-D I(U) \cdot V|=O\left(\|V\|_{E}^{2}\right) .
\end{aligned}
$$

Next we shall prove that $I(U)$ is Fréchet differentiable. For $U, V \in E$,

$$
\begin{aligned}
|I(U+V)-I(U)-D I(U) \cdot V| \\
=\mid \frac{1}{2} \int_{\Omega}(-\Delta U-\Delta V) \cdot(U+V) d x-\int_{\Omega} F(x, U+V) d x \\
\quad-\frac{1}{2} \int_{\Omega}(-\Delta U) \cdot U d x+\int_{\Omega} F(x, U) d x-\int_{\Omega}\left(-\Delta U-F_{U}(x, U)\right) \cdot V d x \mid \\
=\mid \frac{1}{2} \int_{\Omega}[-\Delta U \cdot V-\Delta V \cdot U-\Delta V \cdot V] d x \\
\quad-\int_{\Omega}[F(x, U+V)-F(x, U)] d x-\int_{\Omega}\left[\left(-\Delta U-F_{U}(x, U)\right) \cdot V\right] d x \mid .
\end{aligned}
$$

By (2.2),

$$
\|I(U+V)-I(U)-D I(U) \cdot V\|=O\left(\|V\|_{E}^{2}\right) .
$$

Thus $I \in C^{1}$.

Let $E=W_{0}^{1,2}(\Omega, R) \times \cdots \times W_{0}^{1,2}(\Omega, R)$ and let $\left\{e_{1}, e_{2}, \ldots, e_{n}\right\}$ be an orthonormal basis in $R^{n}$. Then

$$
L^{2}\left(\Omega, R^{n}\right)=\bigoplus_{m \in N} M\left(\lambda_{m}\right)
$$

where $N$ is a natural number and $M\left(\lambda_{m}\right)=\operatorname{span}\left\{\phi_{m} e_{1}, \ldots, \phi_{m} e_{n}\right\}$ is the eigenspace of $-\Delta$ with eigenvalue $\lambda_{m}, \operatorname{dim} M\left(\lambda_{m}\right)=n, m=1,2, \ldots$ Let

$$
L_{1}=\bigoplus_{-\infty<\lambda_{m}<\alpha} M\left(\lambda_{m}\right), \quad L_{0}=\bigoplus_{\alpha<\lambda_{m}<\beta} M\left(\lambda_{m}\right), \quad L_{2}=\bigoplus_{\beta<\lambda_{m}<\infty} M\left(\lambda_{m}\right) .
$$

Then

$$
L^{2}\left(\Omega, R^{n}\right)=L_{1} \oplus L_{0} \oplus L_{2}
$$

For each $X \in L^{2}\left(\Omega, R^{n}\right)$, we have the composition

$$
X=X_{1}+X_{0}+X_{2}
$$

where $X_{1} \in L_{1}, X_{0} \in L_{0}, X_{2} \in L_{2}$. Let $P_{1}$ be the orthogonal projection from $L^{2}\left(\Omega, R^{n}\right)$ onto $L_{1}, P_{0}$ be that from $L^{2}\left(\Omega, R^{n}\right)$ onto $L_{0}$ and $P_{2}$ be that from $L^{2}\left(\Omega, R^{n}\right)$ onto $L_{2}$. Let

$$
V=(-\Delta)^{-1} L_{0}, \quad W_{1}=(-\Delta)^{-1} L_{1}, \quad W_{2}=(-\Delta)^{-1} L_{2}
$$


Then $E=V \oplus W_{1} \oplus W_{2}$, and for $U \in E$, $U$ has the decomposition $U=Y+Z_{1}+Z_{2} \in E$, where

$$
Y=(-\Delta)^{-1} X_{0} \in V, \quad Z_{1}=(-\Delta)^{-1} X_{1} \in W_{1}, \quad Z_{2}=(-\Delta)^{-1} X_{2} \in W_{2} .
$$

Let $W=W_{1} \oplus W_{2}$. Then $W$ is the orthogonal complement of $V$ in $E$. Let $P: E \rightarrow V$ be the orthogonal projection of $E$ onto $V$ and $I-P: E \rightarrow W$ denote that of $E$ onto $W$. Then every element $U \in E$ is expressed by $U=Y+Z, Y=P U, Z=(I-P) U$. Then (1.2) is equivalent to the two systems in the two unknowns $Y$ and $Z$ :

$$
\begin{aligned}
& -\Delta Y=P\left(\operatorname{grad}_{U} F(x, Y+Z)\right) \quad \text { in } \Omega, \\
& -\Delta Z=(I-P)\left(\operatorname{grad}_{U} F(x, Y+Z)\right) \quad \text { in } \Omega, \\
& Y=(0, \ldots, 0), \quad Z=(0, \ldots, 0) \quad \text { on } \partial \Omega .
\end{aligned}
$$

Let $Y \in V$ be fixed and consider the function $h: W_{1} \times W_{2} \rightarrow R$ defined by

$$
h\left(Z_{1}, Z_{2}\right)=I\left(Y+Z_{1}+Z_{2}\right)
$$

The function $h$ has continuous partial Fréchet derivatives $D_{1} h$ and $D_{2} h$ with respect to its first and second variables given by

$$
D_{i} h\left(Z_{1}, Z_{2}\right) \cdot X_{i}=D I\left(Y+Z_{1}+Z_{2}\right) \cdot X_{i}
$$

for $X_{i} \in W_{i}, i=1,2$. By Lemma 2.2, $I$ is a functional of class $C^{1}$.

By the following Lemma 2.3, we can get critical points of the functional $I(U)$ on the infinite dimensional space $E$ from that of the functional on the finite dimensional subspace $V$.

Lemma 2.3 (Reduction lemma) Assume that F satisfies conditions (F1)-(F4). Then

(i) there exists a unique solution $Z \in W$ of the equation

$$
\begin{aligned}
& -\Delta Z=(I-P)\left(\operatorname{grad}_{U} F(x, Y+Z)\right) \text { in } \Omega, \\
& Z=(0, \ldots, 0) \text { on } \partial \Omega .
\end{aligned}
$$

If we put $Z=\Theta(Y)$, then $\Theta$ is continuous on $V$ and satisfies a uniform Lipschitz condition in $V$ with respect to the $L^{2}$ norm (also norm $\|\cdot\|$ ). Moreover,

$$
D I(Y+\Theta(Y)) \cdot X=0 \quad \text { for all } X \in W
$$

(ii) There exists $m_{1}<0$ such that if $Z_{1}$ and $X_{1}$ are in $W_{1}$ and $Z_{2} \in W_{2}$, then

$$
\left(D_{1} h\left(Z_{1}, Z_{2}\right)-D_{1} h\left(X_{1}, Z_{2}\right)\right)\left(Z_{1}-X_{1}\right) \leq m_{1}\left\|Z_{1}-X_{1}\right\|^{2} .
$$

(iii) There exists $m_{2}>0$ such that if $Z_{2}$ and $X_{2}$ are in $W_{2}$ and $Z_{1} \in W_{1}$, then

$$
\left(D_{2} h\left(Z_{1}, Z_{2}\right)-D_{2} h\left(Z_{1}, X_{2}\right)\right) \cdot\left(Z_{2}-X_{2}\right) \geq m_{2}\left\|Z_{2}-X_{2}\right\|^{2} .
$$


(iv) If I I $V \rightarrow R$ is defined by $\tilde{I}(Y)=I(Y+\Theta(Y))$, then I has a continuous Fréchet derivative DI with respect to $Y$, and

$$
D \tilde{I}(Y) \cdot B=D I(Y+\Theta(Y)) \cdot B \text { for all } Y, B \in V \text {. }
$$

(v) $Y_{0} \in V$ is a critical point of I if and only if $Y_{0}+\Theta\left(Y_{0}\right)$ is a critical point of I.

Proof (i) Let $\delta=\frac{\alpha+\beta}{2}$. Equation (2.7) is equivalent to

$$
Z=(-\Delta-\delta)^{-1}(I-P)\left(\operatorname{grad}_{U} F(x, Y+Z)-\delta(Y+Z)\right)
$$

System (2.10) can be rewritten as

$$
\begin{aligned}
& z_{i}=(-\Delta-\delta)^{-1}(I-P)\left(F_{u_{i}}\left(x, y_{1}+z_{1}, \ldots, y_{n}+z_{n}\right)-\delta\left(y_{1}+z_{1}, \ldots, y_{n}+z_{n}\right)\right), \\
& \quad i=1,2, \ldots, n, \\
& y_{1}=\cdots=y_{n}=0, \quad z_{1}=\cdots=z_{n}=0 \quad \text { on } \partial \Omega,
\end{aligned}
$$

where $u_{i}=y_{i}+z_{i}, i=1,2, \ldots, n, U=\left(u_{1}, \ldots, u_{n}\right), Y=\left(y_{1}, \ldots, y_{n}\right), Z=\left(z_{1}, \ldots, z_{n}\right)$. The operator $(-\Delta-\delta)^{-1}(I-P)$ is a self-adjoint, compact and linear map from $(I-P) L^{2}(\Omega, R)$ into itself, and by condition (F3) its $L_{2}$ norm is $\min \left\{\lambda_{h+m+1}-\delta, \delta-\lambda_{h}\right\}^{-1}$. Let $U_{1}, U_{2} \in E$. Since

$$
\begin{aligned}
& \left(F_{u_{i}}\left(x, U_{2}\right)-\delta U_{2}\right)-\left(F_{u_{i}}\left(x, U_{1}\right)-\delta U_{1}\right) \\
& \quad \leq \max \{|\alpha-\delta|,|\beta-\delta|\}\left\|U_{2}-U_{1}\right\|_{R^{n}}=\frac{\alpha+\beta}{2}\left\|U_{2}-U_{1}\right\|_{R^{n}}
\end{aligned}
$$

it follows that the right-hand side of (2.11) defines, for fixed $Y \in V$, a Lipschitz mapping of $(I-P) L^{2}(\Omega, R)$ into itself with Lipschitz constant $r=\left(\min \left\{\lambda_{h+m+1}-\delta, \delta-\lambda_{h}\right\}\right)^{-1} \times \frac{\alpha+\beta}{2}<1$ because $\lambda_{h+m+1}-\delta>\frac{\alpha+\beta}{2}$ and $\delta-\lambda_{h}>\frac{\alpha+\beta}{2}$. Therefore, by the contraction mapping principle, for each given $Y \in V, i=1,2, \ldots, n$, there exists a unique $z_{i} \in(I-P) L^{2}(\Omega, R)$ which satisfies (2.11). Thus, for fixed $Y \in V$, there exists a unique $Z \in(I-P) L^{2}\left(\Omega, R^{n}\right)$ which satisfies (2.10). If $\Theta(Y)$ denotes the unique $Z \in(I-P) L^{2}\left(\Omega, R^{n}\right)$ which solves (2.10), then $\Theta$ is continuous and satisfies a uniform Lipschitz condition in $Y$ with respect to the $L^{2}$ norm (also norm $\left.\|\cdot\|_{E}\right)$. In fact, if $Z_{1}=\Theta\left(Y_{1}\right)$ and $Z_{2}=\Theta\left(Y_{2}\right)$, then

$$
\begin{aligned}
\left\|Z_{1}-Z_{2}\right\|_{L^{2}\left(\Omega, R^{n}\right)}= & \|(-\Delta-\delta)^{-1}(I-P)\left(\operatorname{grad}_{U} F\left(x, Y_{1}+Z_{1}\right)-\delta\left(Y_{1}+Z_{1}\right)\right) \\
& -\left(\operatorname{grad}_{U} F\left(x, Y_{2}+Z_{2}\right)-\delta\left(Y_{2}+Z_{2}\right)\right) \|_{L^{2}\left(\Omega, R^{n}\right)} \\
\leq & r\left\|\left(Y_{1}+Z_{1}\right)-\left(Y_{2}+Z_{2}\right)\right\|_{L^{2}\left(\Omega, R^{n}\right)} \\
\leq & r\left(\left\|Y_{1}-Y_{2}\right\|_{L^{2}\left(\Omega, R^{n}\right)}+\left\|Z_{1}-Z_{2}\right\|_{L^{2}\left(\Omega, R^{n}\right)}\right) \\
\leq & r\left\|Y_{1}-Y_{2}\right\|_{E}+r\left\|Z_{1}-Z_{2}\right\|_{E} .
\end{aligned}
$$

Hence

$$
\left\|Z_{1}-Z_{2}\right\|_{L^{2}\left(\Omega, R^{n}\right)} \leq C\left\|Y_{1}-Y_{2}\right\|_{L^{2}\left(\Omega, R^{n}\right)}, \quad C=\frac{r}{1-r} .
$$


Let $U=Y+Z, Y \in V$ and $Z=\Theta(Y)$. If $X \in(I-P) L^{2}\left(\Omega, R^{n}\right) \cap E$,

$$
\begin{aligned}
D I(Y+\Theta(Y)) \cdot X= & \int_{\Omega}\left[-\Delta(Y+\Theta(Y)) \cdot X-P\left(\left(\operatorname{grad}_{U} F(x, Y+Z)-\delta(Y+Z)\right) \cdot X\right)\right. \\
& \left.-(I-P)\left(\left(\operatorname{grad}_{U} F(x, Y+Z)-\delta(Y+Z)\right) \cdot X\right)\right] d x .
\end{aligned}
$$

It follows from (2.7) that

$$
\int_{\Omega}\left[-\Delta Z(x) \cdot X(x)-\operatorname{grad}_{U} F(x, Y(x)+Z(x)) \cdot X(x)\right] d x=0 .
$$

Since

$$
\int_{\Omega}-\Delta Y(x) \cdot X(x) d x=0
$$

we have

$$
D I(Y+\Theta(Y)) \cdot X=0
$$

(ii) If $Z_{1}$ and $X_{1}$ are in $W_{1}$ and $Z_{2} \in W_{2}$, then

$$
\begin{aligned}
\left(D_{1} h\left(Z_{1}, Z_{2}\right)-D_{1} h\left(X_{1}, Z_{2}\right)\right)\left(Z_{1}-X_{1}\right)= & \int_{\Omega}\left[\left|\nabla\left(Z_{1}-X_{1}\right)\right|^{2}-\left(\operatorname{grad}_{U} F\left(x, Y+Z_{1}+Z_{2}\right)\right.\right. \\
& \left.\left.-\operatorname{grad}_{U} F\left(x, Y+X_{1}+Z_{2}\right)\right) \cdot\left(Z_{1}-X_{1}\right)\right] d x .
\end{aligned}
$$

Since $\int_{\Omega}\left|\nabla\left(Z_{1}-X_{1}\right)\right|^{2}=\left\|Z_{1}-X_{1}\right\|_{E}^{2} \leq \lambda_{h}\left\|Z_{1}-X_{1}\right\|_{L^{2}\left(\Omega, R^{n}\right)}^{2}$ and

$$
\begin{aligned}
& \int_{\Omega}\left(\operatorname{grad}_{U} F\left(x, Y+Z_{1}+Z_{2}\right)-\operatorname{grad}_{U} F\left(x, Y+X_{1}+Z_{2}\right)\right) \cdot\left(Z_{1}-X_{1}\right) \\
& \quad \geq \alpha\left\|Z_{1}-X_{1}\right\|_{L^{2}\left(\Omega, R^{n}\right)} \geq \frac{\alpha}{\lambda_{h}}\left\|Z_{1}-X_{1}\right\|_{E} \\
& \left(D_{1} h\left(Z_{1}, Z_{2}\right)-D_{1} h\left(X_{1}, Z_{2}\right)\right)\left(Z_{1}-X_{1}\right) \leq\left(1-\frac{\alpha}{\lambda_{h}}\right)\left\|Z_{1}-X_{1}\right\|_{E}^{2}
\end{aligned}
$$

where $1-\frac{\alpha}{\lambda_{h}}<0$.

(iii) Similarly, using the fact that $\int_{\Omega}\left|\nabla\left(Z_{2}-X_{2}\right)\right|^{2} d x=\left\|Z_{2}-X_{2}\right\|_{E}^{2} \geq \lambda_{h+m+1} \| Z_{2}-$ $X_{2} \|_{L^{2}\left(\Omega, R^{n}\right)}^{2}$ and

$$
\begin{aligned}
& \int_{\Omega}\left(\operatorname{grad}_{U} F\left(x, Y+Z_{1}+Z_{2}\right)-\operatorname{grad}_{U} F\left(x, Y+Z_{1}+X_{2}\right)\right) \cdot\left(Z_{2}-X_{2}\right) \\
& \quad \leq \beta\left\|Z_{2}-X_{2}\right\|_{L^{2}\left(\Omega, R^{n}\right)} \leq \frac{\beta}{\lambda_{h+m+1}}\left\|Z_{2}-X_{2}\right\|_{E}^{2}
\end{aligned}
$$

we see that if $Z_{2}$ and $X_{2}$ are in $W_{2}$ and $Z_{1} \in W_{1}$, then

$$
\left(D_{2} h\left(Z_{1}, Z_{2}\right)-D_{2} h\left(Z_{1}, X_{2}\right)\right)\left(Z_{2}-X_{2}\right) \geq\left(1-\frac{\beta}{\lambda_{h+m+1}}\right)\left\|Z_{2}-X_{2}\right\|_{E}^{2}
$$

where $\left(1-\frac{\beta}{\lambda_{h+m+1}}\right)>0$. 
(iv) Since the functional $I$ has a continuous Fréchet derivative $D I, \tilde{I}$ has a continuous Fréchet derivative $D \tilde{I}$ with respect to $Y$.

(v) Suppose that there exists $Y_{0} \in V$ such that $D \tilde{I}\left(Y_{0}\right)=0$. From $D \tilde{I}(Y) \cdot B=D I(Y+\Theta(Y))$. $B$ for all $Y, B \in V, D I\left(Y_{0}+\Theta\left(Y_{0}\right)\right)(B)=D \tilde{I}\left(Y_{0}\right)(B)=0$ for all $B \in V$. Since $D I(Y+\Theta(Y)) \cdot B=$ 0 for all $B \in W$ and $E$ is the direct sum of $V$ and $W$, it follows that $D I\left(Y_{0}+\Theta\left(Y_{0}\right)\right)=0$. Thus $Y_{0}+\Theta\left(Y_{0}\right)$ is a solution of (1.1). Conversely, if $U$ is a solution of (1.1) and $Y=P U$, then $D \tilde{I}(Y)=0$.

Remark We note that if $Y \in V$, then $\Theta(Y)=0$.

\section{Proof of Theorem 1.1}

Lemma 3.1 ((PS) condition) Assume that F satisfies conditions (F1)-(F4). Then $-\tilde{I}(v)$ is bounded from below and $\tilde{I}(v)$ satisfies the Palais-Smale condition.

Proof We have

$$
\begin{aligned}
\tilde{I}(Y)= & I\left(Y+\Theta_{1}(Y)+\Theta_{2}(Y)\right) \\
= & \frac{1}{2} \int_{\Omega}\left(-\Delta\left(Y+\Theta_{1}(Y)+\Theta_{2}(Y)\right) \cdot\left(Y+\Theta_{1}(Y)+\Theta_{2}(Y)\right)\right) d x \\
& -\int_{\Omega} F\left(x, Y+\Theta_{1}(Y)+\Theta_{2}(Y)\right) d x \\
= & \frac{1}{2} \int_{\Omega}\left(-\Delta\left(Y+\Theta_{1}(Y)\right) \cdot\left(Y+\Theta_{1}(Y)\right)\right) d x \\
& -\int_{\Omega} F\left(x, Y+\Theta_{1}(Y)\right) d x+\frac{1}{2} \int_{\Omega}\left(-\Delta \Theta_{2}(Y)\right) \cdot \Theta_{2}(Y) d x \\
& -\int_{\Omega}\left[F\left(x, Y+\Theta_{1}(Y)+\Theta_{2}(Y)\right)-F\left(x, Y+\Theta_{1}(Y)\right)\right] d x .
\end{aligned}
$$

We claim that

$$
\begin{aligned}
& \frac{1}{2} \int_{\Omega}\left(-\Delta \Theta_{2}(Y)\right) \cdot \Theta_{2}(Y) d x-\int_{\Omega}\left[F\left(x, Y+\Theta_{1}(Y)+\Theta_{2}(Y)\right)-F\left(x, Y+\Theta_{1}(Y)\right)\right] d x \\
& \quad \leq 0 .
\end{aligned}
$$

In fact, we note that

$$
\begin{aligned}
& \frac{1}{2} \int_{\Omega}\left(-\Delta \Theta_{2}(Y)\right) \cdot \Theta_{2}(Y) d x \\
& \quad=-\frac{1}{2} \int_{\Omega}\left(-\Delta \Theta_{2}(Y)\right) \cdot \Theta_{2}(Y) d x+\int_{\Omega}\left(-\Delta \Theta_{2}(Y)\right) \cdot \Theta_{2}(Y) d x \\
& \quad=-\frac{1}{2} \int_{\Omega}\left(-\Delta \Theta_{2}(Y)\right) \cdot \Theta_{2}(Y) d x+\int_{\Omega} F_{U}\left(x, Y+\Theta_{1}(Y)+\Theta_{2}(Y)\right) \cdot \Theta_{2}(Y) d x
\end{aligned}
$$

by (1.1). We also note that

$$
\begin{aligned}
& F\left(x, Y+\Theta_{1}(Y)+\Theta_{2}(Y)\right)-F\left(x, Y+\Theta_{1}(Y)\right) \\
& \quad=\int_{0}^{1} F_{U}\left(x, Y+\Theta_{1}(Y)+t \Theta_{2}(Y)\right) \cdot \Theta_{2}(Y) d t .
\end{aligned}
$$


Thus we have

$$
\begin{aligned}
\frac{1}{2} \int_{\Omega}( & \left.-\Delta \Theta_{2}(Y)\right) \cdot \Theta_{2}(Y) d x-\int_{\Omega}\left[F\left(x, Y+\Theta_{1}(Y)+\Theta_{2}(Y)\right)-F\left(x, Y+\Theta_{1}(Y)\right)\right] d x \\
= & -\frac{1}{2} \int_{\Omega}\left(-\Delta \Theta_{2}(Y)\right) \cdot \Theta_{2}(Y) d x+\int_{\Omega} F_{U}\left(x, Y+\Theta_{1}(Y)+\Theta_{2}(Y)\right) \cdot \Theta_{2}(Y) d x \\
& -\int_{0}^{1} \int_{\Omega} F_{U}\left(x, Y+\Theta_{1}(Y)+t \Theta_{2}(Y)\right) \cdot \Theta_{2}(Y) d x d t .
\end{aligned}
$$

We note that

$$
\begin{aligned}
\frac{d}{d t}( & \left.F_{U}\left(x, Y+\Theta_{1}(Y)+t \Theta_{2}(Y)\right) \cdot t \Theta_{2}(Y)\right) \\
= & \left(d_{U}^{2} F\left(x, Y+\Theta_{1}(Y)+t \Theta_{2}(Y)\right) \cdot t \Theta_{2}(Y)\right) \cdot \Theta_{2}(Y) \\
& +F_{U}\left(x, Y+\Theta_{1}(Y)+t \Theta_{2}(Y)\right) \cdot \Theta_{2}(Y)
\end{aligned}
$$

which leads to

$$
\begin{aligned}
\int_{0}^{1} & \frac{d}{d t}\left(F_{U}\left(x, Y+\Theta_{1}(Y)+t \Theta_{2}(Y)\right) \cdot t \Theta_{2}(Y)\right) d t \\
= & \int_{0}^{1}\left(d_{U}^{2} F\left(x, Y+\Theta_{1}(Y)+t \Theta_{2}(Y)\right) \cdot t \Theta_{2}(Y)\right) \cdot \Theta_{2}(Y) d t \\
& +\int_{0}^{1} F_{U}\left(x, Y+\Theta_{1}(Y)+t \Theta_{2}(Y)\right) \cdot \Theta_{2}(Y) d t
\end{aligned}
$$

That is,

$$
\begin{aligned}
F_{U}\left(x, Y+\Theta_{1}(Y)+\Theta_{2}(Y)\right) \cdot \Theta_{2}(Y) \\
=\int_{0}^{1}\left(d_{U}^{2} F\left(x, Y+\Theta_{1}(Y)+t \Theta_{2}(Y)\right) \cdot t \Theta_{2}(Y)\right) \cdot \Theta_{2}(Y) d t \\
\quad+\int_{0}^{1} F_{U}\left(x, Y+\Theta_{1}(Y)+t \Theta_{2}(Y)\right) \cdot \Theta_{2}(Y) d t
\end{aligned}
$$

Thus we have

$$
\begin{gathered}
F_{U}\left(x, Y+\Theta_{1}(Y)+\Theta_{2}(Y)\right) \cdot \Theta_{2}(Y)-\int_{0}^{1} F_{U}\left(x, Y+\Theta_{1}(Y)+t \Theta_{2}(Y)\right) \cdot \Theta_{2}(Y) d t \\
=\int_{0}^{1}\left(d_{U}^{2} F\left(x, Y+\Theta_{1}(Y)+t \Theta_{2}(Y)\right) \cdot t \Theta_{2}(Y)\right) \cdot \Theta_{2}(Y) d t .
\end{gathered}
$$

Thus we have

$$
\begin{aligned}
\frac{1}{2} \int_{\Omega}\left(-\Delta \Theta_{2}(Y)\right) \cdot \Theta_{2}(Y) d x-\int_{\Omega}\left[F\left(x, Y+\Theta_{1}(Y)+\Theta_{2}(Y)\right)-F\left(x, Y+\Theta_{1}(Y)\right)\right] d x \\
=-\frac{1}{2} \int_{\Omega}\left(-\Delta \Theta_{2}(Y)\right) \cdot \Theta_{2}(Y) d x+\int_{\Omega} F_{U}\left(x, Y+\Theta_{1}(Y)+\Theta_{2}(Y)\right) \cdot \Theta_{2}(Y) d x \\
\quad-\int_{0}^{1} \int_{\Omega} F_{U}\left(x, Y+\Theta_{1}(Y)+t \Theta_{2}(Y)\right) \cdot \Theta_{2}(Y) d x d t
\end{aligned}
$$




$$
\begin{aligned}
= & -\frac{1}{2} \int_{\Omega}\left(-\Delta \Theta_{2}(Y)\right) \cdot \Theta_{2}(Y) d x \\
& +\int_{0}^{1} \int_{\Omega}\left(d_{U}^{2} F\left(x, Y+\Theta_{1}(Y)+t \Theta_{2}(Y)\right) \cdot t \Theta_{2}(Y)\right) \cdot \Theta_{2}(Y) d x d t \leq 0
\end{aligned}
$$

by condition (F2). Thus by condition (F3) we have

$$
\begin{aligned}
\tilde{I}(Y) & \leq \frac{1}{2} \int_{\Omega}\left(-\Delta\left(Y+\Theta_{1}(Y)\right) \cdot\left(Y+\Theta_{1}(Y)\right)\right) d x-\int_{\Omega} F\left(x, Y+\Theta_{1}(Y)\right) d x \\
& \leq \frac{1}{2}\left(\lambda_{h+m}-\gamma\right)\left\|Y+\Theta_{1}(Y)\right\|_{E}^{2}+C \rightarrow-\infty \text { as }\left\|Y+\Theta_{1}(Y)\right\|_{E} \rightarrow \infty .
\end{aligned}
$$

Thus $-I(\tilde{Y})$ is bounded from below and satisfies the (PS) condition.

Lemma 3.2 Assume that $F$ satisfies conditions (F1)-(F4). Then $Y=\theta, \theta=(0, \ldots, 0)$ is neither minimum nor degenerate.

Proof By (3.1) we have

$$
\tilde{I}(Y) \leq \frac{1}{2} \int_{\Omega}\left(-\Delta\left(Y+\Theta_{1}(Y)\right) \cdot\left(Y+\Theta_{1}(Y)\right)\right) d x-\int_{\Omega} F\left(x, Y+\Theta_{1}(Y)\right) d x
$$

Since

$$
\begin{aligned}
F\left(x, Y+\Theta_{1}(Y)\right) & =\int_{0}^{1} \frac{d F}{d t}\left(x, t\left(Y+\Theta_{1}(Y)\right)\right) d t \\
& =\int_{0}^{1} F_{U}\left(x, t\left(Y+\Theta_{1}(Y)\right)\right) \cdot\left(Y+\Theta_{1}(Y)\right) d t
\end{aligned}
$$

we have that

$$
\begin{aligned}
& \left|\int_{\Omega} F\left(x, Y+\Theta_{1}(Y)\right)-\frac{1}{2} \int_{\Omega} d_{U}^{2} F(x, \theta) \cdot\left(Y+\Theta_{1}(Y)\right) \cdot\left(Y+\Theta_{1}(Y)\right) d x\right| \\
& \quad=\left|\int_{0}^{1} \int_{\Omega}\left[F_{U}\left(x, t\left(Y+\Theta_{1}(Y)\right)\right)-\left(d_{U}^{2} F(x, \theta) \cdot t\left(Y+\Theta_{1}(Y)\right)\right) \cdot\left(Y+\Theta_{1}(Y)\right)\right] d x d t\right| \\
& \quad \leq \frac{1}{2} \sup _{0<t<1}\left\|d_{U}^{2} F\left(x, t\left(Y+\Theta_{1}(Y)\right)\right)-d_{U}^{2} F(x, \theta)\right\|_{\mathcal{L}(V, V)}\left\|Y+\Theta_{1}(Y)\right\|_{E}^{2} .
\end{aligned}
$$

Thus we have

$$
\begin{aligned}
& -\int_{\Omega} F\left(x, Y+\Theta_{1}(Y)\right) \\
& \quad \leq-\frac{1}{2} \int_{\Omega}\left(d_{U}^{2} F(x, \theta) \cdot\left(Y+\Theta_{1}(Y)\right)\right) \cdot\left(Y+\Theta_{1}(Y)\right)+o\left(\left\|Y+\Theta_{1}(Y)\right\|^{2}\right)_{E}
\end{aligned}
$$

Since $\Theta_{1} \in C^{1}\left(V, W_{1}\right)$, it follows that if $\|Y\| \rightarrow 0$, then $\left\|\Theta_{1}(Y)\right\|_{E}=O\left(\|Y\|_{E}\right)$ because $\Theta_{1}(\theta)=\theta$. Thus

$$
\left\|Y+\Theta_{1}(Y)\right\|_{E}=O\left(\|Y\|_{E}\right) .
$$


Since $F_{U}(x, \theta)=\theta$, there exists a bounded self-adjoint operator $A \in \mathcal{L}(E, E)$ which commutes with $P_{o}$ and $P_{1}$ such that

$$
\lambda_{h+1} I \leq A \leq d_{U}^{2} F(x, \theta)
$$

Thus we have

$$
\begin{aligned}
\tilde{I}(Y) \leq & \frac{1}{2} \int_{\Omega}\left(-\Delta\left(Y+\Theta_{1}(Y)\right) \cdot\left(Y+\Theta_{1}(Y)\right)\right) d x \\
& -\frac{1}{2} \int_{\Omega}\left(d_{U}^{2} F(x, \theta) \cdot\left(Y+\Theta_{1}(Y)\right)\right) \cdot\left(Y+\Theta_{1}(Y)\right) d x+o\left(\|Y\|_{E}^{2}\right) \\
\leq & \frac{1}{2} \int_{\Omega}\left(-\Delta\left(Y+\Theta_{1}(Y)\right) \cdot\left(Y+\Theta_{1}(Y)\right)\right) d x \\
& -\frac{1}{2} \int_{\Omega} A\left(Y+\Theta_{1}(Y)\right) \cdot\left(Y+\Theta_{1}(Y)\right)+o\left(\|Y\|_{E}^{2}\right) \\
= & \frac{1}{2} \int_{\Omega}\left(-\Delta \Theta_{1}(Y) \cdot \Theta_{1}(Y)\right) d x-\frac{1}{2} \int_{\Omega} A\left(\Theta_{1}(Y)\right) \cdot \Theta_{1}(Y)+\frac{1}{2} \int_{\Omega}(-\Delta Y \cdot Y) d x \\
& -\frac{1}{2} \int_{\Omega} A(Y) \cdot Y+o\left(\|Y\|_{E}^{2}\right)
\end{aligned}
$$

as $\|Y\|_{E} \rightarrow 0$. Since $\lambda_{h+1} I \leq A$, it follows that

$$
\begin{aligned}
& \frac{1}{2} \int_{\Omega}\left(-\Delta \Theta_{1}(Y) \cdot \Theta_{1}(Y)\right) d x-\frac{1}{2} \int_{\Omega} A\left(\Theta_{1}(Y)\right) \cdot \Theta_{1}(Y) \\
& \quad \leq \frac{1}{2} \int_{\Omega}\left(-\Delta \Theta_{1}(Y) \cdot \Theta_{1}(Y)\right) d x-\frac{1}{2} \int_{\Omega} \lambda_{h+1} \Theta_{1}(Y) \cdot \Theta_{1}(Y) \leq 0 .
\end{aligned}
$$

Therefore we have

$$
\begin{aligned}
\tilde{I}(Y) & \leq \frac{1}{2} \int_{\Omega}(-\Delta Y \cdot Y) d x-\frac{1}{2} \int_{\Omega} A(Y) \cdot Y+o\left(\|Y\|_{E}^{2}\right) \\
& \leq \frac{1}{2} \int_{\Omega}\left[(-\Delta Y) \cdot Y-\lambda_{h+1} Y^{2}\right] d x+o\left(\|Y\|_{E}^{2}\right)
\end{aligned}
$$

as $\|Y\|_{E} \rightarrow 0$. Similarly we can choose a bounded self-adjoint operator $B \in \mathcal{L}(E, E)$ which commutes with $P_{o}$ and $P_{2}$ such that

$$
d_{U}^{2} F(x, \theta) \leq B \leq \lambda_{h+m+1} I
$$

This leads to

$$
\begin{aligned}
\tilde{I}(Y) & \geq \frac{1}{2} \int_{\Omega}(-\Delta Y \cdot Y) d x-\frac{1}{2} \int_{\Omega} B(Y) \cdot Y+o\left(\|Y\|_{E}^{2}\right) \\
& \geq \frac{1}{2} \int_{\Omega}\left[(-\Delta Y) \cdot Y-\lambda_{h+m+1} Y^{2}\right] d x+o\left(\|Y\|_{E}^{2}\right)
\end{aligned}
$$

as $\|Y\|_{E} \rightarrow 0$. Thus $Y=\theta, \theta=(0, \ldots, 0)$ is neither minimum nor degenerate. 
Lemma 3.3 Assume that F satisfies conditions (F1)-(F4). Then

$$
\tilde{I}(Y) \rightarrow-\infty \text { as }\|Y\|_{E} \rightarrow \infty
$$

Proof The proof can be found in the proof of Lemma 3.1.

Proof of Theorem 1.1 By Lemma 2.2, $\tilde{I}(Y)$ is continuous and Fréchet differentiable in $V$. By Lemma 3.1, $-\tilde{I}(Y)$ satisfies the (PS) condition. By Lemma 3.2, $Y=\theta$ is neither minimum nor degenerate. By Lemma 3.3, $\tilde{I}(Y) \rightarrow-\infty$ as $\|Y\|_{E} \rightarrow \infty$. We note that $\max _{Y \in V} \tilde{I}(Y)>0$ is another critical value of $\tilde{I}$. Thus there exists the third critical point of $\tilde{I}(Y)$. Thus (1.1) has at least three nontrivial solutions.

\section{Competing interests}

The authors declare that they have no competing interests.

\section{Authors' contributions}

All authors contributed equally to the manuscript and read and approved the final manuscript.

\section{Author details}

'Department of Mathematics, Kunsan National University, Kunsan, 573-701, Korea. ${ }^{2}$ Department of Mathematics Education, Inha University, Incheon, 402-751, Korea.

\section{Acknowledgements}

This work (Tacksun Jung) was supported by Basic Science Research Program through the National Research Foundation of Korea (NRF) funded by the Ministry of Education, Science and Technology (KRF-2010-0023985).

Received: 21 February 2015 Accepted: 27 February 2015 Published online: 14 March 2015

\section{References}

1. Ha, KS, Lee, YH: Existence of multiple positive solutions of singular boundary value problems. Nonlinear Anal. TMA 28 , 1429-1438 (1997)

2. Lee, YH: Existence of multiple positive radial solutions for a semilinear elliptic system on an unbounded domain. Nonlinear Anal. TMA 47, 3649-3660 (2001)

3. Lee, YH: A multiplicity result of positive radial solutions for a multiparameter elliptic system on an exterior domain. Nonlinear Anal. TMA 45, 597-611 (2001)

4. Lee, YH: Multiplicity of positive radial solutions for multiparameter semilinear elliptic systems on an annulus. J. Differ. Equ. 174, 420-441 (2001)

5. Dunninger, DR, Wang, H: Multiplicity of positive radial solutions for an elliptic system on an annulus domain. Nonlinear Anal. TMA 42(5), 803-811 (2000)

6. Lan, K, Webb, RL: Positive solutions of semilinear equation with singularities. J. Differ. Equ. 148, 407-421 (1998)

7. Chang, KC: Infinite Dimensional Morse Theory and Multiple Solution Problems. Birkhäuser, Basel (1993)

8. Choi, QH, Jung, T: Multiple periodic solutions of a semilinear wave equation at double external resonances. Commun. Appl. Anal. 3(1), 73-84 (1999)

\section{Submit your manuscript to a SpringerOpen ${ }^{\circ}$ journal and benefit from:}

- Convenient online submission

- Rigorous peer review

- Immediate publication on acceptance

- Open access: articles freely available online

- High visibility within the field

- Retaining the copyright to your article

Submit your next manuscript at $>$ springeropen.com 